\title{
Sedation or Anesthesia Before Cervical Cancer Brachytherapy
}

\author{
Antonio Cassio Assis Pellizzon* \\ Department of Radiation Oncology, AC Camargo Cancer Center, Brazil
}

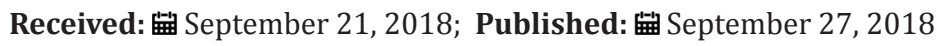

*Corresponding author: Antonio Cassio Assis Pellizzon, Department of Radiation Oncology, AC Camargo Cancer Center, Sao Paulo, Brazil

\begin{abstract}
Cervical cancer (CC) is a neoplasm with great potential for prevention, but it is still a public health problem in most developing countries. There are no significant differences between the use of high dose rate (HDR-BT) and low dose rate (LDR-BT) brachytherapy when considering overall, disease specific and recurrence free survivals. Cervical dilatation is mandatory for the insertion of intrauterine tandems in any of the techniques cited above. Pain and discomfort may eventually be limiting factors of the outpatient procedure and may lead to unsatisfactory results in terms of adequate position of the applicator set. We reviewed the current sedation and anesthetic options for comfort and safety procedures when performing HDR-BT.
\end{abstract}

\section{Introduction}

Cervical cancer (CC) is a neoplasm with great potential for prevention, but it is still an important public health problem in most developing countries, leading to a significant number of deaths in young women (15-50 years old). In 2012 there has been a total of 528,000 new diagnosis and 266,000 deaths per CC worldwide, accounting for $7.5 \%$ of all deaths from female cancers [1]. High dose rate brachytherapy (HDR-BT) with after-loading equipment has been used in the treatment of CC since the 1960s, in radical and also in post-operative settings. HDR-BT has now been widely used due to its mechanical and computerized control evolution besides cost reduction. It presents very satisfactory results in terms local control of disease, depending on clinical stage, when associated or not to External Beam Radiotherapy (EBRT). A literature review published showed no significant differences between HDR-BT and low dose rate brachytherapy (LDR-BT) when considering overall, disease specific and recurrence free survivals. Local control, recurrence and metastasis incidence rates, plus treatment related complications, were not different when comparing both techniques. Some potential advantages of HDR-BT as the rigid immobilization, possibility of outpatient treatment and individualized treatment, are the basis for recommendations of the use of HDR-BT for all clinical stages of CC [2]. Most centers have dedicated HDR services with a significant number of patients treated as outpatients. In these services, outside specialized or general hospitals, and even inside these, the presence of an anesthetic team is not frequent.
In the radical intracavitary technique of HDR-BT one of the most difficulty procedures is the cervical dilatation, necessary for the insertion of the intra-uterine tandem. Despite the tandem relative thinner diameter, when compared to low dose rate tandems, some discomfort is still experienced by patients, even when antispasmodic are prescribed before the procedure. Pain, anxiety and discomfort may be limiting factors of the outpatient procedure, sometimes leading to unsatisfactory results in terms of adequate position of the applicator set [3]. The American Brachytherapy Society recommends that conscious sedation should be used HDRBT insertions whenever possible [4]. Local interventions have been explored to increase patient comfort during HDR-BT. The use of local spray lidocaine was evaluated by Chen et al. and, although considered safe, it was not effective in pain relief [5]. Paracervical block of the cervix is often used as a form of local anesthesia. A systematic review of the various local anesthetic techniques used during outpatient hysteroscopy concluded that paracervical local anesthetic injections are a reasonable method for patients who undergo such procedures but there are doubts if it is appropriate for CC [6]. The use of cervical dilators has been also described but associated to other forms of sedation (opioids and/or paracervical block) [7]. The administration of misoprostol $400 \mu \mathrm{g}$ before tandem insertion was evaluated by Cepni et al. [8] in a randomized trial. The conclusion was that if the medication was taken orally $3 \mathrm{~h}$ before tandem application it facilitates the procedure, increasing patient tolerability and comfort [8]. Lumbar blocks and epidural blockage 
are suitable for inpatients. In Brazil and probably worldwide, besides US and Europe, it is underused due to the shortage of anesthesiologists.

\section{Conclusion}

Few studies have compared the efficacy or toxicity of the various anesthetic methods that can be used for HDR-BT, so it is a wide and open field. Sedation protocols might result in better positioning and or packing. As mentioned previously, each method of sedation and analgesia have advantages and disadvantages, the indication of one or other procedure shall be individualized.

\section{References}

1. Ferlay J, Soerjomataram I, Dikshit R, Eser S, Mathers C, et al. (2015) Cancer incidence and mortality worldwide: sources, methods and major patterns in GLOBOCAN2012. Int J Cancer 136(5): E359-86.

2. Liu R, Wang X, Tian JH, Yang K, Wang J, et al. (2014) High dose rate versus low dose rate intracavity brachytherapy for locally advanced uterine cervix cancer. Cochrane Database Syst Rev Oct 7(7): CD007563.
3. Pellizzon ACA (2008) Braquiterapia de alta taxa de dose: tumores ginecológicos. In: Pellizzon, ACA et al. Rotinas e condutas em radioterapia. ( $3^{\text {rd }}$ edn.) São Paulo: Lemar 3: 80-87.

4. Nag S, Erickson B, Thomadsen B, Demanes JD, Petereit D, et al. (2000) The American Brachytherapy Society recommendations for high-doserate brachytherapy for carcinoma of the cervix. Int J Radiat Oncol Biol Phys 48(1): 201-211.

5. Chen HC, Leung SW, Wang CJ, Sun LM, Fang FM, et al. (1998) Local vaginal anesthesia during high-dose-rate intracavitary brachytherapy for cervical cancer. Int J Radiat Oncol Biol Phys 42(3): 541-544.

6. Cooper NA, Khan KS, Clark TJ (2010) Local anesthesia for pain control during outpatient hysteroscopy: systematic review and meta-analysis. BMJ 340: c1130.

7. Mayr NA, Sorosky JI, Zhen W, Weidner GJ, Hussey DH, et al. (1998) The use of laminarias for osmotic dilation of the cervix in gynecological brachytherapy applications. Int J Radiat Oncol Biol Phys 42(5): 10491053.

8. Cepni K, Gul S, Cepni I, Güralp O, Sal V, et al. (2011) Randomized trial of oral misoprostol treatment for cervical ripening before tandem application in cervix cancer. Int J Radiat Oncol Biol Phys 81(3): 778-781.

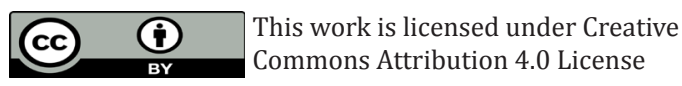

To Submit Your Article Click Here: Submit Article

DOI: 10.32474/IGWHC.2018.02.000146

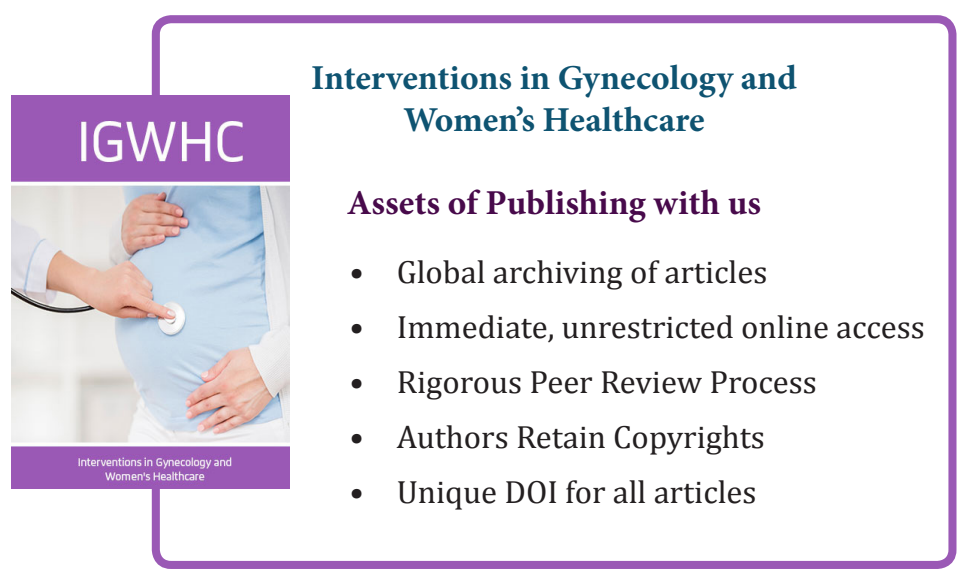

